\title{
INGESTA DE SAL YODADA EN HOGARES Y ESTADO NUTRICIONAL DE YODO EN MUJERES EN EDAD FÉRTIL EN PERÚ, 2008
}

\author{
Ana María Higa ${ }^{1, a}$, Marianella Miranda ${ }^{2, a}$, Miguel Campos 3 ,b,c, José R. Sánchez 2,a
}

\begin{abstract}
RESUMEN
Objetivo. Estimar el estado nutricional de yodo en mujeres en edad fértil y evaluar la ingesta de sal adecuadamente yodada en sus hogares en el Perú. Materiales y métodos. Estudio transversal realizado con un muestreo probabilístico multietápico por conglomerados que incluyó 1573 hogares y 2048 mujeres en edad fértil, distribuidas en cinco dominios (Lima, resto de costa, sierra rural, sierra urbana y selva). Se evaluó el uso de sal yodada en hogares y el yodo urinario en mujeres en edad fértil. Resultados. El 97,5\% (IC95\%: 96,7 - 98,5\%) de hogares peruanos consumen sal yodada, siendo menor en sierra rural (95\%) y mayor en Lima metropolitana (100\%). La mediana de yoduria nacional fue de 266 $\mu \mathrm{g} / \mathrm{L}$, siendo menores en la selva $(206 \mu \mathrm{g} / \mathrm{L})$ y mayores en el resto de costa $(302 \mu \mathrm{g} / \mathrm{L})$, estos valores se encuentran por encima del límite recomendado por la Organización Mundial de la Salud en todos los dominios (mediana mayor a $200 \mu \mathrm{g} / \mathrm{L}$ ), valor que asegura un control de desórdenes por deficiencia de yodo (DDI). Conclusiones. En control de los DDI por el consumo de sal yodado es bueno, se debe continuar con la vigilancia de la presencia de yodo en la sal, particularmente en las áreas con menor acceso.
\end{abstract}

Palabras clave: Yodo; Deficiencia de yodo; Niveles de yodo urinario; Encuestas nutricionales; Perú (fuente: DeCS BIREME).

\section{IODIZED SALT INTAKE IN HOUSEHOLDS AND IODINE NUTRITIONAL STATUS IN WOMEN OF CHILDBEARING AGE IN PERU, 2008}

\begin{abstract}
Objective. To estimate the iodine nutritional status in women of childbearing age and to evaluate the intake of salt adequately iodized in their households in Peru. Materials and methods. Cross-sectional study performed with a multistage, probabilistic, cluster sampling that included 1573 households and 2048 women in childbearing age, distributed in five domains (Lima, rest of the coast, rural highlands and jungle area). The use of iodized salt was evaluated in the households and the urinary iodine was evaluated in the childbearing age women. Results. $97.5 \%(95 \% \mathrm{Cl}$. 96.7-98.5\%) of peruvian households have iodized salt consumption, being it lower in the rural highland (95\%) and higher in Lima $(100 \%)$. The national mean of urinary iodine was $266 \mu \mathrm{g} / \mathrm{L}$, being it lower in the jungle areas $(206 \mu \mathrm{g} / \mathrm{L})$ and higher in the rest of the coast $(302 \mu \mathrm{g} / \mathrm{L})$, these values are above the level recommended by the World Health Organization in all domains (average higher than $200 \mu \mathrm{g} / \mathrm{L}$ ), value that ensures control over the iodine deficiency disorders (IDD). Conclusions. Control of IDD associated with iodized salt consumption is good, and monitoring of the presence of iodine in salt, particularly in the areas with lowest access, should continue.
\end{abstract}

Key words: lodo; lodine deficiency; lodo, urinary levels; Nutrition survey; Peru (source: MeSH NLM).

\section{INTRODUCCIÓN}

El yodo es un micronutriente esencial en la formación de las hormonas tiroideas, cuya función principal es regular el crecimiento y desarrollo físico e intelectual del individuo; su deficiencia durante la gestación y en los primeros años de vida produce daño cerebral irreversible y disminución de la capacidad intelectual de los niños ${ }^{(1)}$. La deficiencia de yodo es uno de los principales problemas de salud pública mundial que afecta a gestantes y niños pequeños, se expresa en una mayor mortalidad perinatal y diversos grados de retardo mental; actualmente se reconoce que los desórdenes por deficiencia de yodo (DDI) son la principal causa prevenible del daño cerebral infantil, siendo esta la razón central que motiva la cruzada mundial de eliminarla como problema de salud pública (2).

La principal causa del problema es la baja ingesta de yodo ${ }^{(3)}$. Se reconoce una condición natural geográfica presente en las zonas montañosas y las inundables, que han sufrido los efectos de la glaciación y erosión permanentes, procesos que han lavado el yodo de los suelos y lo han derivado al lecho marino ${ }^{(4)}$. Como resultado de ello, los alimentos y el agua de estas regiones contienen muy escasa cantidad de yodo y por tanto las dietas basadas en los productos locales tienen muy poca concentración de este nutriente ${ }^{(5,6)}$.

\footnotetext{
Departamento Académico de Nutrición, Facultad de Medicina, Universidad Nacional Mayor de San Marcos. Lima, Perú.

2 Dirección Ejecutiva de Vigilancia Alimentaria Nutricional, Centro Nacional de Alimentación y Nutrición, Instituto Nacional de Salud. Lima, Perú.

3 Departamento de Física, Informática y Matemáticas, Facultad de Ciencias y Filosofía, Universidad Peruana Cayetano Heredia. Lima, Perú.
}

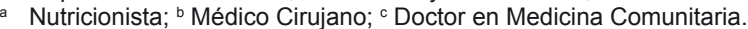


Esta situación es aplicable a nuestras regiones de sierra y selva, por lo que, aun cuando el problema se ha controlado, las poblaciones residentes en dichas zonas están en riesgo permanente. Por ello, es fundamental continuar la intervención con sal yodada de manera sostenida, para garantizar una adecuada ingesta y alcanzar la nutrición óptima de yodo ${ }^{(7)}$.

En el Perú, la deficiencia de yodo se ha reconocido desde el siglo XIX, pero ha sido enfrentada exitosamente recién en las últimas décadas mediante un programa nacional de prevención y control, implementado por el Ministerio de Salud de Perú (MINSA) desde 1986 y que fue diseñado con el objetivo de lograr el control de la deficiencia de yodo antes del año 2000. Este plan incluyó una combinación de intervenciones en función a la situación basal: la organización de una red de trabajo nacional capacitada dentro de las Direcciones Regionales de Salud, alianzas con otros agentes sociales; el tratamiento masivo con suplementos de aceite yodado a las poblaciones de mayor riesgo y la fortificación de la sal con yodo como alternativa universal sostenible ${ }^{(5)}$.

Asimismo, se desarrolló el sistema de vigilancia epidemiológica en base al monitoreo de la sal yodada y la evaluación del estado nutricional del yodo en la población en riesgo a los DDI, mediante la medición de la concentración de yodo urinario en escolares. El modelo aplicado permitió lograr la meta de eliminación virtual de los DDI en 1998, y ofrecer condiciones de sostenibilidad para mantener su vigilancia, lo cual fue certificado por Organización Panamericana de la Salud (OPS)/Organización Mundial de la Salud OMS- el Fondo Internacional de las Naciones Unidas de Auxilio a la Infancia (UNICEF de su nombre en inglés) y el Consejo Internacional para el Control de la Deficiencia de Yodo (ICCIDD de su nombre en inglés), siendo confirmado nuevamente en el año $2004{ }^{(8)}$.

Luego, el MINSA con la cooperación técnica y financiera del UNICEF y la asesoría del ICCIDD, ha continuado las intervenciones básicas de monitoreo y vigilancia de la yodación de la sal y del estado nutricional del yodo en la población, según las normas establecidas. Estas acciones han permitido sostener el control de los DDI y avanzar hacia la nutrición óptima de yodo hasta la fecha ${ }^{(9)}$.

Con el objetivo de asegurar la sostenibilidad del control de los DDI en el Perú, el Centro Nacional de Alimentación y Nutrición del Instituto Nacional de Salud (CENAN, INS), adicionalmente al seguimiento en escolares en las áreas de riesgo de DDI, ha incorporado dentro del sistema de monitoreo nacional de indicadores nutricionales (MONIN), el seguimiento de la ingesta de sal yodada en el hogar y del estado nutricional de yodo mediante evaluación de yodurias en mujeres en edad fértil (MEF) y niños menores de 5 años. El objetivo de este estudio fue evaluar el estado nutricional de yodo en mujeres en edad fértil y evaluar la ingesta de sal adecuadamente yodada en sus hogares en el Perú.

\section{MATERIALES Y MÉTODOS}

\section{DISEÑO DEL ESTUDIO}

Se realizó un estudio observacional transversal, el MONIN es una encuesta nacional con poder de inferencia a nivel nacional y en cinco estratos (Lima metropolitana, resto de costa, sierra urbana, sierra rural y selva) para la estimación de indicadores de impacto, determinantes y procesos de la desnutrición infantil. El muestreo realizado es probabilístico continuo, estratificado y polietápico.

Para este estudio, se usaron los datos recabados a nivel nacional en dos periodos realizados entre el 19 de noviembre de 2007 y el 2 de marzo de 2008 (periodo 2008-I), y entre el 27 de junio de 2008 y el 5 de octubre de 2008 (periodo 2008-II).

\section{POBLACIÓN Y MUESTRA}

Se estudiaron 1753 hogares de niños menores de 5 años y 2048 MEF quienes fueron seleccionadas de esos mismos hogares. La distribución por ámbitos es la siguiente: Lima Metropolitana 331, Resto de Costa 342, Sierra Urbana 315, Sierra Rural 351 y Selva 414.

El cálculo del tamaño muestral, se estimó tomando como base los valores promedio de prevalencia de las variables a estudiar, asumiendo una tasa de rechazo o pérdida no mayor del $15 \%$, un efecto de diseño del orden 1,50; un nivel de confianza de $95 \%$ y con un margen de error de 7,5 a $10 \%$.

Se incluyeron a mujeres entre 15 a 49 años de edad, que no estén convalecientes de alguna enfermedad u operación, que no tengan una medicación de yodo por problemas tiroideos, que no estén menstruando o tomando suplemento de sulfato ferroso o que la muestra de orina tenga apariencia de contaminación por secreciones densas o cuerpos extraños.

\section{PROCEDIMIENTOS}

Para evaluar la ingesta de sal yodada, se solicitó en cada hogar entrevistado, una cucharadita de sal de la que empleaba para preparar sus alimentos, al que se le agregó una gota del reactivo Yoditest y luego de 
observar el color resultante de la reacción y compararlo con la escala de color, se anotaba la alternativa respectiva $(0,7,15$ y $>30 \mathrm{ppm})$. Adicionalmente en el grupo correspondiente al periodo 2008 III, se colectó muestras de sal de los hogares, en envases de polietileno específicamente destinadas para tal fin, para medir la concentración de yodo por el método cuantitativo.

Asimismo, se recolectó una muestra casual de orina de una MEF en cada hogar, en un frasco de polietileno debidamente rotulado y sellado, luego fueron enviadas conservadas en refrigeración al laboratorio de CENAN, INS en Lima.

Para la determinación de yodo urinario se usó el método de amonio persulfato, basado en la reacción Sandell Kolthoff, modificado de Pino y Dunn ${ }^{(10,11)}$ y para el análisis cuantitativo de yodo en sal se utilizó el método volumétrico ${ }^{(12)}$; la lectura de la concentración de yodo en ambos casos, se realizó por espectrofotometría.

\section{ANALISIS DE DATOS}

Para el análisis de resultados de ingesta de sal yodada, se consideró el porcentaje de muestras positivas al reactivo cualitativo, equivalente a 7 o más ppm; teniendo en cuenta lo recomendado por el grupo de expertos OMS-UNICEF-ICCIDD ${ }^{(11)}$.

Para evaluar el estado nutricional de yodo, se consideró el valor de la concentración de yodo en orina, $\geq 100 \mu \mathrm{g} / \mathrm{L}$ , siguiendo lo recomendado por OMS ${ }^{(11,12)}$.

La información fue analizada con el paquete estadístico R 2.10.1 usando el módulo Survey ${ }^{(13,14)}$. El análisis de yoduria se realizó en el total de la muestra, 2048 mujeres en edad fértil, incluyendo 77 gestantes. Se calculó la proporción e intervalo de confianza al 95\% de hogares que consumen sal yodada, así como la mediana de yoduria, tanto a nivel nacional como por dominio.

\section{RESULTADOS}

La información obtenida de la evaluación de ingesta, yodo positivo equivalente a $\geq 7$ ppm, mostró que 97,5 \% de hogares consumían sal yodada, con pequeñas variaciones entre dominios, siendo el de menor ingesta la sierra rural, 95\% y el de mayor ingesta Lima Metropolitana con 100\%; seguido muy de cerca por sierra urbana, 99,7\%. (Tabla 1).

Estos resultados han sido confirmados con determinación cuantitativa de yodo en sal realizada en 616 muestras recogidas de los hogares encuestados en el periodo 2008 III. El promedio de yodación en esta muestra fue de $31,7 \mathrm{ppm} \pm, 13,6$ y la mediana fue de 34,5 ppm (P20: $18,5$ y $\mathrm{P} 80: 41,8)$.

Con relación al estado nutricional de yodo (Tabla 2), en mujeres en edad fértil, se encontró que en todos los dominios de estudio la mediana de yoduria fue superior a $200 \mu \mathrm{g} / \mathrm{L}$ con variaciones de $206 \mu \mathrm{g} / \mathrm{L}$ en la selva a $302 \mu \mathrm{g} / \mathrm{L}$ en Resto de Costa, siendo la mediana nacional de $266 \mu \mathrm{g} / \mathrm{L}$. Se encontró igualmente que el menor valor en el percentil 20 fue $69 \mu \mathrm{g} / \mathrm{L}$ en la sierra rural; mientras que en percentil 80 , todos los ámbitos estuvieron por encima de $400 \mu \mathrm{g} / \mathrm{L}$. La concentración de yodo urinario se puede visualizar mejor en la Figura 1

Con respecto a la distribución de los valores individuales según rangos, se tuvo los siguientes resultados: $7,5 \%$ menor a $50 \mu \mathrm{g} / \mathrm{L}, 10,2 \%$ entre $50-99 \mu \mathrm{g} / \mathrm{L}, 20,3 \%$ entre $100-199 \mu \mathrm{g} / \mathrm{L}, 19,1 \%$ entre $200-299 \mu \mathrm{g} / \mathrm{L}$ y $41,9 \%$ mayor a $300 \mu \mathrm{g} / \mathrm{L}$. El histograma de frecuencias de yoduria

Tabla 1. Proporción de hogares con ingesta de sal con presencia de yodo equivalente a $\geq 7 \mathrm{ppm}$ y $\geq 15 \mathrm{ppm}$ a nivel nacional y según dominios, Perú 2008*.

\begin{tabular}{|c|c|c|c|c|c|}
\hline \multirow{2}{*}{ Dominio } & \multirow{2}{*}{$\mathbf{N}$} & \multicolumn{2}{|c|}{ Yodo positivo $\geq 7 p^{p} m^{*}$} & \multicolumn{2}{|c|}{ Yodo positivo $\geq 15$ ppm* } \\
\hline & & Proporción & IC $95 \%$ & Proporción & IC $95 \%$ \\
\hline Lima Metropolitana & 331 & 100,0 & $100,0-100,0$ & 92,5 & $88,8-96,7$ \\
\hline Resto Costa & 342 & 97,8 & $96,6-99,1$ & 83,2 & $74,7-91,6$ \\
\hline Sierra Urbana & 315 & 99,7 & $99,4-100,0$ & 92,1 & $87,8-96,3$ \\
\hline Sierra Rural & 351 & 95,0 & $92,0-98,1$ & 79,0 & $67,0-91,0$ \\
\hline Selva & 414 & 96,0 & $94,2-97,8$ & 79,5 & $71,0-87,9$ \\
\hline Nacional & 1753 & 97,5 & $96,7-98,5$ & 85,0 & $81,0-89,0$ \\
\hline
\end{tabular}

* Evaluado por método cualitativo. ppm: partículas por millón. 
Tabla 2. Niveles de yodo en orina $(\mu \mathrm{g} / \mathrm{L})$ en mujeres en edad fértil, según ámbitos.

\begin{tabular}{lccccccc}
\hline Dominio & N & Mediana & IC 95\% & P20 & P80 & Mínimo & Máximo \\
\hline Lima Metropolitana & 587 & 284 & $246-311$ & 142 & 429 & 1 & 13130 \\
Resto Costa & 448 & 302 & $246-383$ & 143 & 514 & 29 & 4303 \\
Sierra Urbana & 257 & 265 & $244-294$ & 155 & 437 & 28 & 1251 \\
Sierra Rural & 498 & 227 & $159-311$ & 69 & 463 & 5 & 2338 \\
Selva & 298 & 206 & $158-\mathbf{3 0 2}$ & 96 & 438 & 9 & 1256 \\
Nacional & $\mathbf{2 0 4 8}$ & $\mathbf{2 6 6}$ & $\mathbf{2 3 2 - \mathbf { 2 9 2 }}$ & $\mathbf{1 1 7}$ & $\mathbf{4 5 7}$ & $\mathbf{1}$ & $\mathbf{1 3} \mathbf{1 3 0}$ \\
\hline
\end{tabular}

P20: percentil 20; p80: percentil 80.

muestra que los datos están sesgados hacia la derecha, lo que corroboraría un porcentaje de yodurias muy altas, como puede observarse en la Figura 2.

\section{DISCUSIÓN}

Los resultados de este estudio, indican que la cobertura que la ingesta de sal yodada en las mujeres en edad fértil es universal, la población utiliza sal embolsada que reacciona positivamente al examen cualitativo de yodo en más del $95 \%$ de los hogares (punto de corte equivalente a $\geq 7 \mathrm{ppm}$ ). Se está considerando sólo el resultado positivo al reactivo, en atención a lo recomendado por el grupo de expertos de OMS, 2007, para el uso del método de análisis cualitativo de yodo en sal ${ }^{(12)}$.

La determinación cuantitativa de yodo en sal realizada en 616 muestras recogidas de los hogares encuestados en el periodo 2008 III, ha mostrado que la concentración mediana de yodo esta en el rango mínimo establecido por Ley a nivel de producción (30 a 40ppm).



Figura 1. Concentración de yodo urinario en edad fértil según ámbitos, Perú 2008.
Al corte equivalente de $\geq 15$ ppm (concentración de yodo para considerar sal adecuadamente yodada a nivel hogar), podríamos afirmar que se mantiene en las áreas urbanas de la costa y sierra en más de $92 \%$, mientras que en el área rural y la selva ha disminuido significativamente (79\%), respecto a lo reportado en años previos ${ }^{(15)}$. Esta disminución podría estar explicada por la disminución en la intensidad de la supervisión de la yodación de sal a nivel de la producción y su comercialización en las zonas más dispersas. La incorporación de mediciones cuantitativas de yodo en sal en muestras de hogares de manera sistemática fortalecerían los mecanismos de control en los productores locales por lo que se recomendaría colectar muestras de sal de una submuestra de hogares ${ }^{(12)}$.

En relación con los niveles de concentración de yodo urinario en las mujeres en edad fértil, se confirma que la deficiencia de yodo no es un problema de salud pública, pues en todos los ámbitos se presenta una mediana de yoduria mayor a $200 \mu \mathrm{g} / \mathrm{L}$ con una variación entre 206 a $302 \mu \mathrm{g} / \mathrm{L}$ siendo la mediana nacional, $266 \mu \mathrm{g} / \mathrm{L}$ Incluso el valor más bajo en el percentil 20 fue de $69 \mu \mathrm{g} / \mathrm{L}$, ubicado

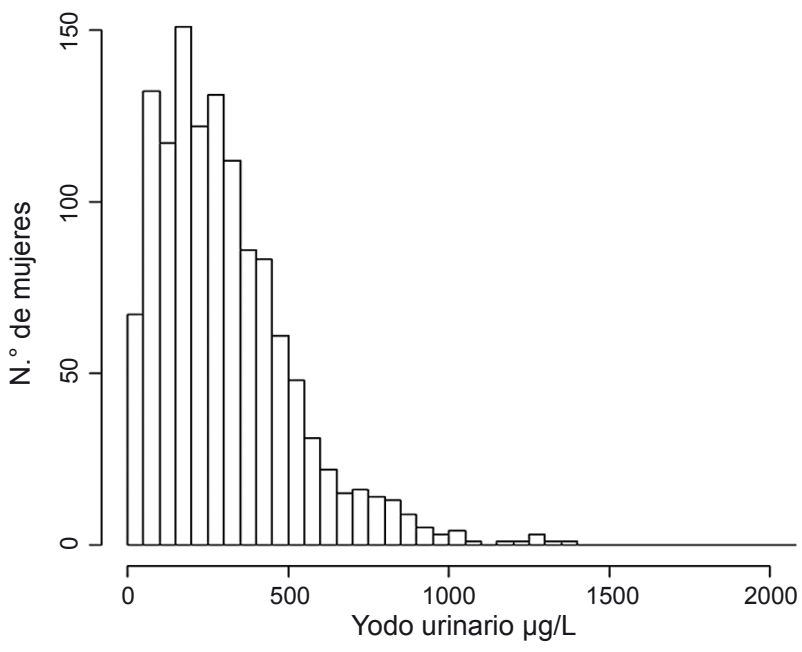

Figura 2. Histograma de frecuencia de yoduria en mujeres en edad fértil, Perú 2008. 
en sierra rural, siendo superior al mínimo sugerido por OMS de $50 \mu \mathrm{g} / \mathrm{L}$. Estos valores son similares a lo encontrado en el mismo grupo de estudio realizado por el MONIN hasta el 2001, y en la encuesta nacional de consumo de alimentos ENCA 2003, realizados también por CENAN ${ }^{(15,16)}$. Aunque es un grupo diferente, el último estudio realizado por el CENAN en escolares en el 2006 (datos no publicados) mostró una mediana de $259 \mu \mathrm{g} / \mathrm{L}$, e ingesta de sal yodada del 96,5\%, muy similar a lo encontrado por el MINSA en 1997, con lo cual puede afirmarse que se mantiene el control de los DDI en Perú $(17,18)$. Según el grupo de Expertos de OMS/UNICEF/ ICCIDD, 2007, la nutrición óptima de yodo se alcanza cuando la mediana de los niveles de yodo en orina se encuentra entre 100 a $200 \mu \mathrm{g} / \mathrm{L}$ y una mediana entre 200 a $299 \mu \mathrm{g} / \mathrm{L}$ sugiere una ingesta adecuada inclusive en gestantes y mujeres que dan de lactar.

Sharmanov et al. (19) en el estudio nacional de micronutrientes en Kazakhstan realizado en el 2006, encontró que las mujeres en edad reproductiva presentaron una mediana de yoduria de $250 \mu \mathrm{g} / \mathrm{L}$ y un ingesta de sal adecuadamente yodada de 92\%; un análisis más detallado de esa encuesta nacional mostró una correlación cercana entre el nivel de ingesta de sal adecuadamente yodada( $\geq$ de $15 \mathrm{ppm}$ ) y la concentración urinaria de yodo $\geq 100 \mu \mathrm{g} / \mathrm{L}$. El estudio NHANES 20032004 de Estados Unidos ha mostrado valores adecuados en MEF mediana de $131 \mu \mathrm{g} / \mathrm{L}^{(20)}$; los cuales se mantienen desde el estudio de 1988; según la referencia, es producto de las medidas que se adoptaron para reducir el exceso de yodo experimentado en la década de los 70-80 en dicho país, atribuido al incremento de ingesta de productos industrializados que aportan importantes cantidades de yodo, además que el nivel de yodación de la sal en dicho país es mayor a 40 ppm.

Según señala OMS en su informe técnico 2007, con los progresos globales rápidos en corregir la deficiencia de yodo se están reconociendo también ejemplos de exceso de yodo particularmente donde la concentración de yodo como fortificante es alta y está siendo mal monitorizada. Se señala allí, que el hombre muestra en general una notable tolerancia frente a las ingestas elevadas de yodo; sin embargo, la situación es muy distinta cuando en la población, previamente, ha existido una deficiencia sustancial de yodo, en especial en personas mayores de 40 años. En estos casos, si la ingesta de yodo aumenta debido a los programas de prevención de los DDI, existe el riesgo de que se produzca una tirotoxicosis ${ }^{(12)}$. Indica el informe también que esta condición adversa podría ocurrir después de 5 a 10 años de intervención con sal yodada, pasado este tiempo, valores de mediana superiores a $300 \mu \mathrm{g} / \mathrm{L}$ no han mostrado efectos colaterales; por ello es importante seguir vigilando el estado de nutrición de yodo. Revisando los datos individuales en nuestro estudio, el $41 \%$ de las muestras están por encima de $300 \mu \mathrm{g} / \mathrm{L}$ lo cual indicaría que como país estaríamos entrando a una etapa en que la ingesta de yodo estaría en un rango entre 300 a 400 ug/día, valor por encima de lo recomendado por OMS; sin embargo, debe mencionarse que la mayor dispersión de los valores de yoduria, encontrados aun en la sierra rural, zona que presentaba deficiencia moderada de yodo hace 25 años atrás ${ }^{(12)}$ indicaría la necesidad de seguir vigilando la adecuada provisión de yodo a través de la sal yodada en dicha zona.

De otro lado, el porcentaje de mujeres que han presentado yoduria mayor a $300 \mu \mathrm{g} / \mathrm{L}$, no tendría explicación sólo por la ingesta de sal yodada, dado que con el promedio de yodo encontrado de 31,7 ppm y la mediana de 34,5 ppm, se esperaría concentraciones de yodo en orina menores; lo que nos llevaría a reflexionar que los valores de yoduria reportados, podría estar relacionada con una mayor diversidad en la dieta que incluiría alimentos procesados o fortificados con mezclas de micronutrientes que incluyen yodo o el añadido de sal yodada en el procesamiento, por lo que es un aspecto que debe investigarse.

La industrialización de alimentos estaría incrementando los aportes dietéticos de yodo, según se viene reportando en algunos países, tal es el caso de Holanda y Dinamarca, donde se ha identificado el pan como una fuente importante de yodo (21), asimismo, en Chile ${ }^{(22)}$. En Eslovenia, un estudio de ingesta de yodo en adolescentes jóvenes mujeres, evaluada mediante encuestas de frecuencia de ingesta y recordatorio de 3 días en una submuestra, reportó una prevalencia de ingesta de $92 \%$ de sal yodada $\geq 15 \mathrm{ppm}$ de yodo, con una mediana de ingesta de 155,8 ug/dia y, en un $11 \%$ de ellas, la ingesta de yodo fue mayor a 300 ug/día. La sal yodada contribuyó con el 39\% de la ingesta promedio/día, las bebidas, con $22 \%$ y leche y derivados con $19 \%{ }^{(23)}$.

En conclusión, el estado nutricional actual del yodo en la MEF es más que adecuado estando las medianas de yoduria de los ámbitos de estudio en un rango entre 204 a $301 \mu \mathrm{g} / \mathrm{L}$, por consiguiente la deficiencia de yodo en Perú no es un problema de salud pública y los nuevos niños que nacerán, estarían potencialmente protegidos de los DDI.

Sin embargo, dadas las diferencias encontradas en área urbana y rural, debiera reforzarse el monitoreo del proceso de yodación de la sal en los pequeños productores que están abasteciendo la sierra rural y la selva. También debiera ser motivo de estudio los valores elevados de yodo urinario en un grupo importante de MEF, recomendándose realizar estudios específicos de consumo de alimentos para investigar otras fuentes de yodo adicionales a la sal 
yodada; estos resultados contribuirían a prever que una eventual disminución de ingesta de sal, no afectaría la provisión adecuada de yodo en la población.

Debe remarcarse que la fortificación de la sal con yodo sigue siendo la medida universalmente recomendada por OMS-UNICEF- ICCIDD ${ }^{(24)}$ para la prevención y control de la deficiencia de yodo, por su mayor costo beneficio por lo que debe continuarse la promoción de su ingesta así como la vigilancia de la calidad de la yodación de la sal a los productores.

\section{AGRADECIMIENTOS}

A la MSc. Ivonne Bernui por su valioso apoyo en la revisión del artículo.

\section{Fuente de Financiamiento}

El estudio fue financiado con recursos ordinarios por el Centro Nacional de Alimentación y Nutrición del Instituto Nacional de Salud, Lima, Perú.

\section{Conflictos de Interés}

Los autores declaran no tener conflicto de interés para la publicación del presente artículo.

\section{REFERENCIAS BIBLIOGRÁFICAS}

1. Hetzel B. Introduction: The nature and magnitude of the iodine deficiency disorders. En: Hetzel B (Editor), Towards the global elimination of brain damage due to iodine deficiency. New Dheli: Oxford University Press; 2004.

2. De Benoist B, Anderson M, Egli I, Takkouche B, Allen H. lodine status worldwide, WHO Global Database on lodine Deficiency. Geneva, World Health Organization; 2004.

3. Delange F. The disorders induced by iodine deficiency. Thyroid. 1994; 4(1): 107-28.

4. Johnson CC, Fordyce FM, Stewart AG. Environmental controls in iodine deficiency disorders. Project summary report. Nottingham: British Geological Survey; 2003.

5. Pretell EA. Desórdenes por deficiencia de yodo (DDI). Generalidades y situación en el Perú. En: Blanco de Alvarado T, Gonzales Mugaburu L. Situación nutricional en el Perú. Lima: Ministerio de Salud/ Organización Panamericana de la Salud; 1989.

6. Cárdenas H, Gomez C, Pretell E. Contenido de yodo en leche de vacuno procedente de la sierra y costa de Perú . ALAN. 2000; 53(4): 408-12.

7. Dunn JT. Sustaining optimal iodine nutrition. En: Hetzel B (Editor). Towards the global elimination of brain damage due to iodine deficiency. New Dheli, Oxford University Press; 2004.

8. World Health Organization. Progress towards the elimination of iodine deficiency disorders (IDD). Geneva: WHO; 1994.

9. PretellEA, Higa AM. Eliminación sostenida de los desórdenes por deficiencia de yodo en Perú. 25 años de experiencia. Acta Med Peru. 2008; 25(3): 128-34.
10. Pino S, Fang SL, Braverman LE. Ammonium persulfate: a safe alternative oxidizing reagent for measuring urinary iodine. Clin Chem. 1996; 42(2): 239-243.

11. Dunn JT, Crutchfield HE, Gutekuntz R, Dunn AD. Methods for measurement iodine in urine. The Netherlands: International Council for control of iodine deficiency disorders ICCIDD- UNICEF-WHO; 1993.

12. World Health Organization. Assessment of the iodine deficiency disorders and monitoring their elimination. 3a Ed. Geneva: WHO; 2007.

13. $R$ Foundation for Statistical Computing, Development Core Team. R: A language and environment for statistical computing. Vienna, R Foundation for Statistical Computing; 2009.

14. Lumley T. Analysis of complex survey samples. Seattle: University of Washington; 2004.

15. Instituto Nacional de Salud, Centro Nacional de Alimentación y Nutrición. Informes técnicos Monitoreo Nacional de Indicadores Nutricionales. 2001, 2004- 2005, 2008. [Documento en línea] [Fecha de acceso: 10 de febrero de 2010] Disponible en: http://www.ins.gob.pe/insvirtual/ins/ cenan/monin/historica_monin.asp

16. Miranda M, Calderón M, Riega V, Barboza J, Rojas C. Ingesta de sal fortificada con yodo en niños de 12 a 35 meses de edad y mujeres en edad fértil en el Peru. Rev Peru Med Exp Salud Publica 2004; 21(2): 82-86.

17. Perú, Ministerio de Salud. Control de la deficiencia de yodo en el Perú. Un modelo sostenible, Programa Nacional de Prevención de Deficiencia de Micronutrientes. Lima: MINSA; 1998.

18. Pretell EA, Delange F, Hostalek U, Corigliano S, Barreda L, Higa AM, et al. lodine nutrition improves in Latin America. Thyroid. 2004, 14(8): 590-99.

19. SharmanovT, Tsoy I, Tazhibajeb S, Kulmurzayeva L, Ospanova F, Karsybekova N, et al. IDD elimination through universal salt iodization in Kazakhstan. IDD Newsl. 2008; 28(1): 4-8.

20. Pearce E. US lodine nutrition: where do we stand? Thyroid 2008; 18(11): 1143-44.

21. Gerasimow G. Increasing iodine intakes in population through the use of iodized salt in bread baking. IDD Newsl. 2009; 33(3): 10-12.

22. Leiva L, Braverman L, Muzzo S. Aporte del pan a la nutrición de yodo del escolar chileno. Rev Chil Nutr. 2002; 29(1): 62-64.

23. Stimec M, Mis NF, Smole K, Sirca-Campa A, Kotnik P, Zupancicl. M, et al lodine intake of Slovenian adolescents. Ann Nutr Metab. 2007; 51(5): 439-47.

24. World Health Organization. Salt as a vehicle for fortification. Geneva: WHO; 2008.

Correspondencia: Marianella Y. Miranda Cuadros. Dirección: Av. Tizón y Bueno N. 276, Lima 11, Perú Teléfono: (511) 6176200 anexos 6605-6636

Correo electrónico: mmiranda@ins.gob.pe 